\title{
Flow-mediated slowing of brachial-radial pulse wave velocity: Methodological aspects and clinical determinants
}

\author{
Nicholas Cauwenberghs, Yenthel Heyrman, Lutgarde Thijs, \\ Wen-Yi Yang, Fang-Fei Wei, Zhen-Yu Zhang, Jan A. Staessen, \\ Tatiana Kuznetsova*
}

\begin{abstract}
Research Unit Hypertension and Cardiovascular Epidemiology, KU Leuven Department of Cardiovascular Sciences, University of Leuven, Belgium
\end{abstract}

Received 17 November 2017; accepted 7 December 2017

Available online 9 January 2018

\section{KEYWORDS}

General population; Endothelial function; Flow-mediated slowing;

Pulse wave velocity

\begin{abstract}
Background: Recent studies proposed that deceleration in pulse wave velocity (PWV) following reactive hyperaemia might reflect arterial distensibility and endothelial function. We therefore investigated methodological aspects and clinical determinants of new indexes of flow-mediated slowing (FMS) of PWV in a community-based sample.

Methods: In 71 subjects (mean age, 60.3 years; $50.7 \%$ women), we continuously assessed brachial-radial PWV using Vicorder ${ }^{\circledR}$ at baseline and after 3-min or 5-min suprasystolic upper-arm cuff occlusion. We calculated the relative change $(\Delta)$ in PWV per each $30 \mathrm{~s}$ intervals during $4 \mathrm{~min}$ of post-occlusion. We performed stepwise regression analyses to assess determinants of the PWV response.

Results: The peak FMS was detected at the first PWV recording obtained after occlusion. Overall, the decline in PWV during hyperaemia was significantly greater after 5-min of occlusion as compared to $3-\mathrm{min}$ (effect sizes for $0-240 \mathrm{~s}$ intervals: $-1.83 \%$ to $-9.63 \% ; P \leq 0.037$ ). PWV declined significantly less with higher age during the $0-60 \mathrm{~s}$ post-occlusion intervals $(P \leq 0.0053)$. On the other hand, after $120 \mathrm{~s}$ of post-occlusion, $\Delta \mathrm{PWV}$ remained significantly lower in subjects with high diastolic blood pressure and oxidized LDL, and in smokers $(P \leq 0.028)$. Consequently, as compared to healthy reference group, participants with cardiovascular risk factors exhibited a delay in age-adjusted recovery of PWV after 5-min of occlusion $(P \leq 0.039)$.

Conclusions: Our findings confirm the use of a 5-min occlusion time for the assessment of vasomotor function by FMS. Whereas the early FMS response might deteriorate with ageing,
\end{abstract}

\footnotetext{
* Corresponding author. Research Unit of Hypertension and Cardiovascular Epidemiology, KU Leuven Department of Cardiovascular Sciences, University of Leuven, Campus Sint Rafaël, Kapucijnenvoer 35, Block D, Box 7001, B-3000 Leuven, Belgium.

E-mail address: tatiana.kouznetsova@med.kuleuven.be (T. Kuznetsova).
} 
cardiovascular risk factors such as smoking, oxidative stress and hypertension might affect recovery of PWV after reactive hyperaemia.

(C) 2017 Association for Research into Arterial Structure and Physiology. Published by Elsevier B.V. All rights reserved.

\section{Introduction}

The vascular endothelium regulates key processes involving platelet function, inflammation, smooth muscle cell proliferation and vascular tone. ${ }^{1}$ Endothelial dysfunction, characterized by reduced nitric oxide (NO) bioavailability, contributes to the progression of cardiovascular diseases such as atherosclerosis and hypertension. ${ }^{2,3}$

The vasodilatory response to increased shear stress during reactive hyperaemia following a brief period of ischaemia is partly triggered by endothelium-dependent release of NO. ${ }^{4}$ This adaptation in vascular tone could be non-invasively assessed by the flow-mediated dilation (FMD) of large arteries, ${ }^{5,6}$ or by the changes in digital pulse wave amplitude. ${ }^{4,7-10}$

Another novel approach to assess the conduit artery vasomotor function is based on changes in brachial-radial pulse wave velocity (PWV) during reactive hyperaemia. ${ }^{11,12}$ The observed decline in PWV during the vasodilation after ischaemia might be used as a marker of arterial distensibility and endothelial function. ${ }^{11,12}$ Indeed, PWV is inversely related to arterial distensibility ${ }^{13,14}$ and reflects in some degree dynamic changes in vascular tone. ${ }^{15}$ Moreover, the assessment of such flow-mediated slowing (FMS) of PWV has emerged as a user-friendly and reproducible alternative to measurement of FMD. ${ }^{12}$ The automated FMS measurement protocol requires little training and logistics, favouring its use in large-scale population studies and screening programs.

To date, however, no study assessed the correlates of the newly proposed FMS index. Therefore, we investigated in a random community-based sample to what extent methodological aspects of FMS measurement and clinical characteristics might affect the temporal decline in brachial-radial PWV following reactive hyperaemia.

\section{Materials and methods}

\section{Study participants}

From August 1985 until December 2005, we randomly recruited a family-based population sample stratified by sex and age from a geographically defined area in northern Belgium, as described previously. ${ }^{10}$ The initial participation rate was $\mathbf{7 8 . 0 \%}$. The Flemish Study on Environment, Genes and Health Outcomes (FLEMENGHO) study is an ongoing population study, in which participants are repeatedly examined at a local examination centre.

From February 2016 until January 2017, a scheduled follow-up examination at the field centre included assessment of endothelial function using an automated FMS protocol. ${ }^{12}$ From 93 invited participants, we obtained informed written consent from 82 subjects (response rate $88.2 \%$ ). Of these, 6 subjects did not undergo the FMS protocol because of advanced age ( $>80$ years). We also excluded 4 participants with frequent extrasystoles $(n=3)$ or a pacemaker $(n=1)$. Furthermore, we discarded one recording of insufficient quality to reliably assess the FMS index. In total, 71 participants were statistically analysed.

\section{FMS protocol}

The participants refrained from smoking, heavy exercise, and drinking alcohol or caffeine-containing beverages for at least 3 hours before the test. We studied FMS in an airconditioned room at constant temperature around $22^{\circ} \mathrm{C}$. To attain a cardiovascular steady-state before starting the test, the subjects had rested for at least 20 min in the supine position.

We implemented an automated FMS protocol using the Vicorder ${ }^{\circledR}$ device (Skidmore Medical, Bristol, UK) (Fig. 1). Participants were lying with their left arm positioned $70-80^{\circ}$ to their body. Two oscillometric cuffs connected to the Vicorder ${ }^{\circledR}$ were placed around the upper arm and wrist. We measured the brachial-radial distance (D) as the distance between the midst of both cuffs. Next, subjects underwent an FMS procedure that included a baseline period of $3 \mathrm{~min}$, an occlusion period of $3 \mathrm{~min}$ or $5 \mathrm{~min}$ in which the brachial cuff was inflated to $200 \mathrm{~mm} \mathrm{Hg}$, and a 4 min post-

\section{A) Brachial-Radial PWV $=D / \Delta T$}

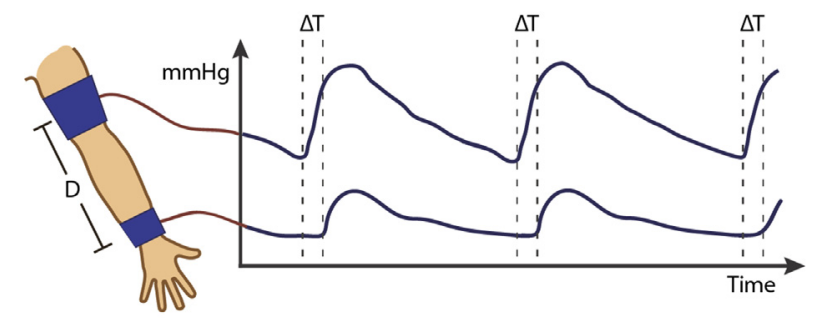

B) FMS Protocol

\begin{tabular}{|c|c|c|}
\hline Baseline & Brachial Occlusion & Reactive Hyperemia \\
\hline » $3 \mathrm{~min}$ & » $3 \min (n=35)$ & $» 4 \mathrm{~min}$ \\
\hline » $P W V_{B L}$ & $» 5 \min (n=36)$ & $\begin{array}{c}\text { "PWV } W_{\text {FMs }} \\
\text { (mean per } 30 \text { s intervals) }\end{array}$ \\
\hline
\end{tabular}

Figure 1 Automated assessment of flow-mediated slowing following reactive hyperaemia. (A) Mild inflation of brachial and radial cuff allow real-time recording of brachial-radial pulse wave velocity. (B) The FMS response equals the relative changes in pulse wave velocity following hyperaemia as induced by suprasystolic brachial occlusion. The FMS response was monitored for 4 min after 3-min or 5-min of occlusion. BL indicates baseline; $D$, brachial-radial distance; $\Delta T$, time difference between brachial and radial pulse upstroke; FMS, flowmediated slowing; PWV, pulse wave velocity. 
occlusion period. During pre- and post-occlusion periods, both cuffs were inflated mildly $(\leq 65 \mathrm{~mm} \mathrm{Hg})$ to simultaneously record brachial and radial pulse wave tracings. The onset-time of upstroke was derived from both the brachial $\left(t_{\text {brachial }}\right)$ and radial pulse tracings $\left(t_{\text {radial }}\right)$ in real-time. The brachial-radial PWV was calculated from these tracing every 3-4 s during the baseline and post-occlusion period as the brachial-radial distance divided by the transit time $\Delta \mathrm{T}\left(\mathrm{PWV}=\mathrm{D} /\left[\mathrm{t}_{\text {radial }}-\mathrm{t}_{\text {brachial }}\right]=\mathrm{D} / \Delta \mathrm{T}\right)$.

We calculated the mean baseline PWV $\left(\mathrm{PWV}_{\mathrm{BL}}\right)$ and mean post-occlusion PWV for each $30 \mathrm{~s}$ interval starting from the first measurement after occlusion (recorded about $15 \mathrm{~s}$ after brachial cuff release). We calculated FMS as the relative change $(\Delta)$ in PWV following hyperaemia (e.g. $\left.\mathrm{FMS}_{0-30}=\left[\mathrm{PWV}_{0-30}-\mathrm{PWV}_{\mathrm{BL}}\right] / \mathrm{PWV}_{\mathrm{BL}} \times 100 \%\right)$.

\section{Other measurements}

Trained study nurses administered a standardized questionnaire to collect detailed information on the participant's medical history, smoking and drinking habits. Hypertension was defined as a blood pressure (BP) of at least $140 \mathrm{~mm} \mathrm{Hg}$ systolic or $90 \mathrm{~mm} \mathrm{Hg}$ diastolic (average of 5 consecutive auscultatory readings at the examination centre) or the use of antihypertensive drugs. Body mass index was weight in kilograms divided by the square of height in meters.

\section{Statistical analysis}

For database management and statistical analysis, we used SAS software, version 9.4 (SAS Institute, Cary, North Carolina, USA). Normality of distributions was evaluated by Shapiro-Wilk's and skewness statistics. We compared means and proportions by a sample $t$-test and chi-square test, respectively. Statistical significance was a two-sided significance level of $P<0.05$.

We performed forward stepwise regression to assess the determinants of FMS during each $30 \mathrm{~s}$ post-occlusion interval. Covariables considered in the stepwise models were age, occlusion time, heart rate, sex, current smoking, body mass index, systolic BP, diastolic BP, mean arterial pressure, pulse pressure, antihypertensive and lipid-lowering drug treatment, history of diabetes mellitus, triglycerides, total cholesterol, high-density (HDL) and low-density lipoprotein (LDL) and oxidized LDL. We set the $P$ values for variables to enter and to stay in the stepwise multiple regression models at 0.10 , but considered a two-sided $P$ value below 0.05 as statistically significant. We assessed the interaction of the FMS response throughout postocclusion periods with the occlusion time, sex and age groups as implemented in PROC GLM.

\section{Results}

\section{Clinical characteristics of participants}

The 71 participants included $36(50.7 \%)$ women. Of the 44 (62.0\%) hypertensive subjects, 25 (56.8\%) were on antihypertensive drug treatment. Table 1 lists the clinical characteristics of the study participants by sex and occlusion time. Compared to women, men had on average higher body mass index, diastolic BP and mean arterial pressure $(P \leq 0.042)$, but lower heart rate, total cholesterol and HDL $(P \leq 0.018)$. The prevalence of hypertension was significantly higher in men than in women $(P=0.0020)$. Clinical characteristics did not differ between subjects who underwent either 3-min or 5-min of upper-arm occlusion $(P \geq 0.089$ for all; Table 1$)$.

\section{Baseline PWV, its change during reactive hyperaemia and FMS}

Table 2 presents the average brachial-radial PWV in absolute values at baseline and by each $30 \mathrm{~s}$ post-deflation interval by sex and occlusion time. At baseline, PWV did not differ significantly between both genders $(P=0.080)$ nor between subjects who underwent the 3 -min or 5 -min occlusion protocol $(P=0.59)$. From the first post-occlusion recording of PWV until $210 \mathrm{~s}$ thereafter, absolute values of PWV were significantly lower in women as compared to men ( $P \leq 0.044$ for all; Table 2$)$. Compared to the 3 -min occlusion period, subjects who underwent 5-min of occlusion had a significantly lower PWV during the $30-90$ s postocclusion intervals $(P \leq 0.042)$.

Figure 2 illustrates the distribution of FMS values by each post-occlusion time interval. Table 3 lists FMS at each time interval during reactive hyperaemia by sex and occlusion time period. Regardless of sex and occlusion time, the peak FMS of PWV was detected at the first PWV recording obtained just after cuff deflation (Table 3; Fig. 3, panel $A$ ). In the $0-180$ s intervals, FMS response was greater in subjects who underwent 5-min of occlusion as compared to 3-min $(P \leq 0.0099$; Table 3; Fig. 3, panel $A)$. Throughout the whole post-occlusion period, the relative decrease in PWV did not differ between men and women ( $P \geq 0.059$; Table 3; Fig. 3, panel $B$ ).

\section{Determinants of FMS}

We performed stepwise regression analyses to assess the determinants of FMS recorded immediately after cuff deflation and during each $30 \mathrm{~s}$ interval of the post-occlusion period (Table 4). First, we observed that the decline in PWV following reactive hyperaemia was significantly greater after 5-min of occlusion as compared to 3-min (effect sizes for $0-240$ s intervals: $-1.83 \%$ to $-9.63 \%$; $P \leq 0.037$ ). While adjusted for occlusion time, PWV declined significantly less with age during the $0-60 \mathrm{~s}$ post-occlusion intervals $(P \leq 0.0053$; Table 4; Fig. 4). Women showed a greater hyperaemia-mediated decrease in PWV than men only during the 60-90 s interval $(-4.00 \% ; P=0.037)$.

We noticed that after $120 \mathrm{~s}$ of post-deflation, adjusted $\triangle \mathrm{PWV}$ remained significantly lower in subjects with high diastolic blood pressure and oxidized LDL, and in smokers $(P \leq 0.028$ for all; Table 4$)$.

\section{FMS and cardiovascular risk factors}

Figure 5 shows the unadjusted and age-adjusted FMS for each $30 \mathrm{~s}$ post-deflation time interval in subjects with 
Table 1 Clinical characteristics of 71 participants by sex and occlusion time.

\begin{tabular}{|c|c|c|c|c|c|c|}
\hline \multirow[t]{2}{*}{ Characteristics } & \multicolumn{3}{|l|}{ Gender } & \multicolumn{3}{|l|}{ Occlusion Time } \\
\hline & Men $(n=35)$ & Women $(n=36)$ & $P$ value & $3 \min (n=35)$ & $5 \min (n=36)$ & $P$ value \\
\hline \multicolumn{7}{|l|}{ Anthropometrics } \\
\hline Age, y & $60.2 \pm 15.3$ & $60.3 \pm 12.1$ & 0.97 & $59.0 \pm 13.4$ & $61.5 \pm 14.0$ & 0.45 \\
\hline Female, n (\%) & - & - & - & $18(51.4)$ & $18(50.0)$ & 0.90 \\
\hline Body mass index, $\mathrm{kg} / \mathrm{m}^{2}$ & $28.4 \pm 3.93$ & $26.3 \pm 4.58$ & 0.042 & $27.5 \pm 4.15$ & $27.2 \pm 4.63$ & 0.77 \\
\hline Systolic BP, mm Hg & $133.2 \pm 10.6$ & $129.9 \pm 16.7$ & 0.33 & $133.0 \pm 11.9$ & $130.1 \pm 15.9$ & 0.39 \\
\hline Diastolic BP, mm Hg & $87.6 \pm 9.55$ & $80.4 \pm 8.22$ & 0.0011 & $85.9 \pm 9.45$ & $82.1 \pm 9.38$ & 0.089 \\
\hline Pulse pressure, $\mathrm{mm} \mathrm{Hg}$ & $45.5 \pm 10.6$ & $49.4 \pm 13.0$ & 0.17 & $47.0 \pm 7.89$ & $48.0 \pm 15.0$ & 0.74 \\
\hline $\mathrm{MAP}, \mathrm{mm} \mathrm{Hg}$ & $102.8 \pm 8.57$ & $96.9 \pm 10.0$ & 0.010 & $101.6 \pm 9.65$ & $98.1 \pm 9.63$ & 0.13 \\
\hline Heart rate, bpm & $60.8 \pm 6.80$ & $65.2 \pm 8.42$ & 0.018 & $63.0 \pm 7.13$ & $63.1 \pm 8.74$ & 0.96 \\
\hline \multicolumn{7}{|l|}{ Questionnaire data } \\
\hline Current smoking, n (\%) & $3(8.6)$ & 7 (19.4) & 0.19 & $3(8.6)$ & $7(19.4)$ & 0.19 \\
\hline Drinking alcohol, n (\%) & $11(31.4)$ & $8(22.2)$ & 0.38 & $7(20.0)$ & $12(33.3)$ & 0.20 \\
\hline Hypertensive, n (\%) & $28(80.0)$ & $16(44.4)$ & 0.0020 & $21(60.0)$ & $23(63.9)$ & 0.74 \\
\hline $\begin{array}{l}\text { Treated for hypertension, } \\
\mathrm{n}(\%)\end{array}$ & $16(45.7)$ & $9(25.0)$ & 0.068 & $10(28.6)$ & $15(41.7)$ & 0.25 \\
\hline$\beta$-blockers, n (\%) & $5(14.3)$ & $3(8.3)$ & 0.43 & $4(11.4)$ & $4(11.1)$ & 0.97 \\
\hline ACE, $n(\%)$ & $5(14.3)$ & $4(11.1)$ & 0.69 & $4(11.4)$ & $5(13.9)$ & 0.76 \\
\hline$A R B, n(\%)$ & $4(11.4)$ & $0(0.0)$ & 0.037 & $3(8.6)$ & $1(2.8)$ & 0.29 \\
\hline $\mathrm{CCB}, \mathrm{n}(\%)$ & $6(17.1)$ & $1(2.8)$ & 0.042 & $3(8.6)$ & $4(11.1)$ & 0.72 \\
\hline Diuretics, n (\%) & $8(22.9)$ & $4(11.1)$ & 0.19 & $5(14.3)$ & $7(19.4)$ & 0.56 \\
\hline Lipid-lowering drugs & $12(34.3)$ & $9(25.0)$ & 0.39 & $8(22.9)$ & $13(36.1)$ & 0.22 \\
\hline History of CHD & $2(5.71)$ & $0(0.0)$ & 0.15 & $1(2.86)$ & $1(2.78)$ & 0.98 \\
\hline History of diabetes & $5(14.3)$ & $5(13.9)$ & 0.96 & $6(17.1)$ & $4(11.1)$ & 0.47 \\
\hline \multicolumn{7}{|l|}{ Biochemical data } \\
\hline Triglycerides, mmol/L & $1.85 \pm 0.86$ & $1.48 \pm 0.64$ & 0.051 & $1.70 \pm 0.80$ & $1.61 \pm 0.75$ & 0.63 \\
\hline Total cholesterol, mmol/L & $4.52 \pm 0.89$ & $5.28 \pm 1.12$ & 0.0027 & $5.02 \pm 1.23$ & $4.80 \pm 0.91$ & 0.42 \\
\hline $\mathrm{HDL}, \mathrm{mmol} / \mathrm{L}$ & $1.31 \pm 0.34$ & $1.78 \pm 0.45$ & $<0.0001$ & $1.45 \pm 0.41$ & $1.66 \pm 0.49$ & 0.066 \\
\hline $\mathrm{LDL}, \mathrm{mmol} / \mathrm{L}$ & $2.58 \pm 0.75$ & $3.00 \pm 1.08$ & 0.066 & $2.99 \pm 1.08$ & $2.60 \pm 0.77$ & 0.092 \\
\hline Oxidized LDL, mU/mL & $314.6(37.0-1003)$ & $382.2(37.0-1200)$ & 0.32 & $309.9(37.0-1003)$ & $388.8(71.0-1164)$ & 0.15 \\
\hline
\end{tabular}

Values are mean $( \pm S D)$, number of subjects $(\%)$ or median (10-90\% percentile). ACE indicates angiotensin-converting enzyme; ARB, angiotensin receptor blockers; BP, blood pressure; CCB, calcium channel blockers; CHD, coronary heart disease; HDL, high-density lipoprotein; LDL, low-density lipoprotein; MAP, mean arterial pressure.

cardiovascular risk factors (hypertension, smoking, diabetes and history of coronary heart diseases) and in healthy reference group. After adjustment for age, we did not observe any differences in the peak hyperaemia-mediated FMS of PWV between subjects with and without risk factors. On the other hand, after 5-min of occlusion, 26 subjects with cardiovascular risk factors exhibited a significant delay in recovery of PWV as compared to 10 healthy subjects $(P \leq 0.039$ for $150-240$ s post-occlusion intervals; Fig. 5). We did not observe any significant difference in FMS between participants with and without CV risk factors who underwent 3 -min of occlusion ( $P \geq 0.27$ for all 30 s postocclusion intervals; Fig. 5).

\section{Discussion}

In this population study, we investigated methodological aspects and clinical determinants of the temporal decline in PWV triggered by reactive hyperaemia. The key findings of our study can be summarized as follows: (i) we detected an overall greater PWV change after a 5-min than after a 3-min occlusion period; (ii) advanced age blunted the peak hyperaemic PWV decline detected after cuff deflation; and (iii) subjects with cardiovascular risk factors exhibited a delay in recovery of PWV as compared to a healthy reference group.

Nitric oxide (NO) is an important endothelium-derived vasodilator with anti-atherosclerotic properties. ${ }^{1}$ The reduction in NO bioavailability, a key feature of endothelial dysfunction, impairs the capacity of the arterial wall to dampen increased shear stress following hyperaemia. ${ }^{4}$ Currently, endothelial function can be assessed noninvasively during reactive hyperaemia using the FMD of the brachial artery, ${ }^{5,6}$ or the change in digital pulse amplitude measured by applanation tonometry $4,7,8$ or photoplethysmography. ${ }^{9,10}$ To date, FMD is considered the gold standard for assessment of vasomotor function in the conduit arteries. However, the technically challenging FMD procedure requires extensive training and standardization to guarantee acceptable reproducibility. ${ }^{5,16}$

Recent studies proposed that flow-mediated changes in PWV during hyperaemic response might be used as a userfriendly, reliable alternative to assess vasomotor 
Table 2 Brachial-radial pulse wave velocity during the FMS protocol by sex and occlusion time.

\begin{tabular}{|c|c|c|c|c|c|c|}
\hline \multirow[t]{2}{*}{ Component } & \multicolumn{3}{|l|}{ Gender } & \multicolumn{3}{|l|}{ Occlusion time } \\
\hline & Men $(\mathrm{n}=35)$ & Women $(n=36)$ & $P$ value & $3 \min (n=35)$ & $5 \min (n=36)$ & $P$ value \\
\hline Baseline PWV (m/s) & $8.37 \pm 1.07$ & $7.83 \pm 1.48$ & 0.080 & $8.01 \pm 1.20$ & $8.18 \pm 1.43$ & 0.59 \\
\hline \multicolumn{7}{|c|}{ PWV $(\mathrm{m} / \mathrm{s})$ by time intervals $(\mathrm{s})^{*}$} \\
\hline First measurement & $7.27 \pm 1.20$ & $6.61 \pm 1.27$ & 0.028 & $7.21 \pm 1.20$ & $6.68 \pm 1.30$ & 0.080 \\
\hline $0-30$ & $7.58 \pm 1.19$ & $6.88 \pm 1.31$ & 0.021 & $7.51 \pm 1.25$ & $6.95 \pm 1.29$ & 0.065 \\
\hline $30-60$ & $7.83 \pm 1.28$ & $7.13 \pm 1.34$ & 0.028 & $7.81 \pm 1.34$ & $7.15 \pm 1.30$ & 0.037 \\
\hline $60-90$ & $7.95 \pm 1.06$ & $7.03 \pm 1.31$ & 0.0019 & $7.80 \pm 1.28$ & $7.18 \pm 1.20$ & 0.042 \\
\hline $90-120$ & $7.97 \pm 0.83$ & $7.35 \pm 1.26$ & 0.018 & $7.80 \pm 1.15$ & $7.52 \pm 1.06$ & 0.30 \\
\hline $120-150$ & $8.06 \pm 0.80$ & $7.44 \pm 1.27$ & 0.017 & $7.88 \pm 1.19$ & $7.62 \pm 1.01$ & 0.31 \\
\hline $150-180$ & $8.06 \pm 0.89$ & $7.53 \pm 1.26$ & 0.044 & $7.94 \pm 1.19$ & $7.66 \pm 1.04$ & 0.30 \\
\hline $180-210$ & $7.95 \pm 0.99$ & $7.40 \pm 1.10$ & 0.030 & $7.72 \pm 1.20$ & $7.63 \pm 0.96$ & 0.74 \\
\hline $210-240$ & $8.06 \pm 0.90$ & $7.58 \pm 1.33$ & 0.085 & $7.88 \pm 1.20$ & $7.76 \pm 1.12$ & 0.66 \\
\hline
\end{tabular}

Values are mean $( \pm S D)$. *Time intervals were relative to the first PWV recording measured just after cuff deflation. FMS indicates flowmediated slowing; PWV, pulse wave velocity.
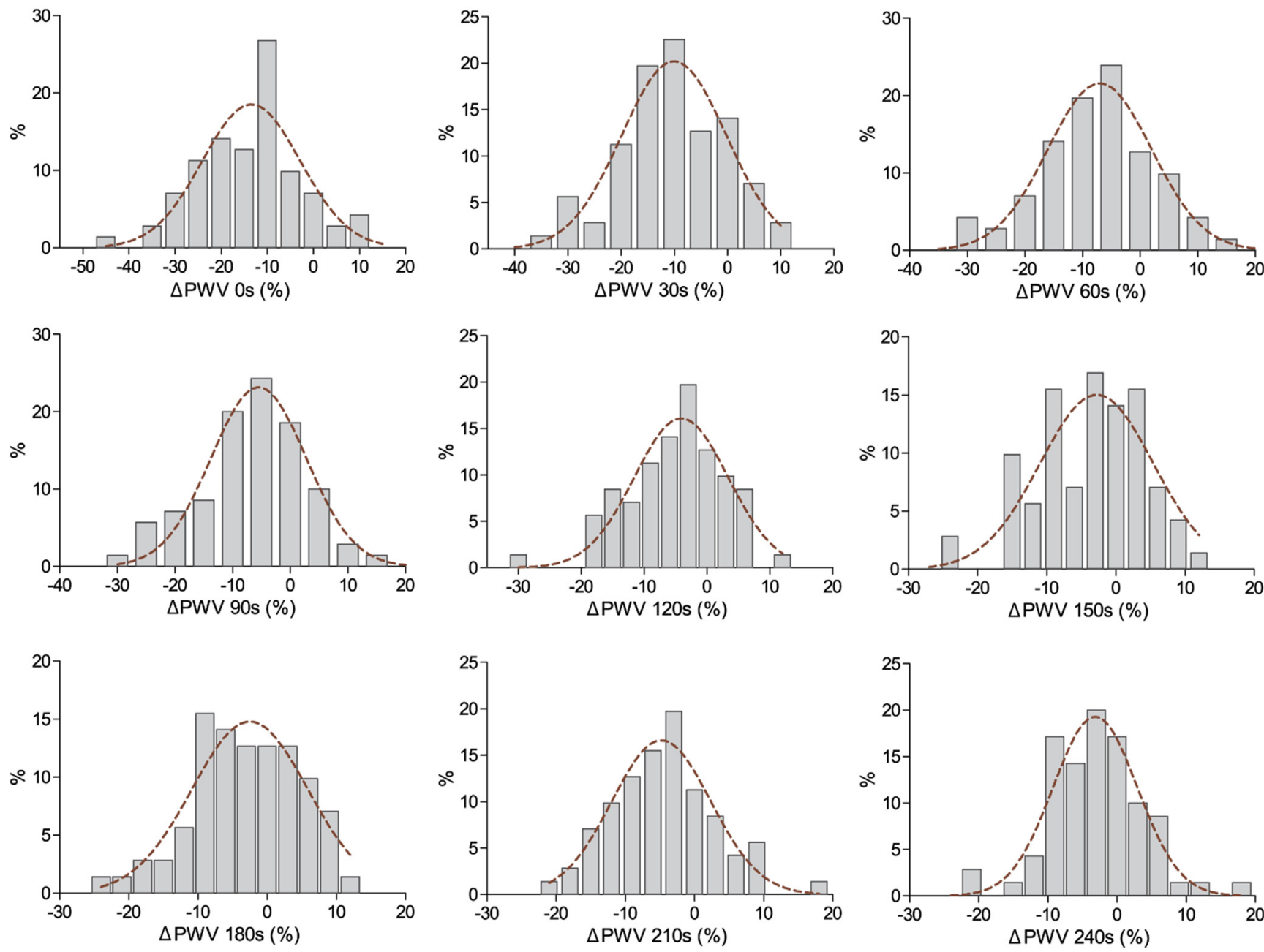

Figure 2 Distributions of hyperaemia-mediated changes in pulse wave velocity $(\Delta \mathrm{PWV})$ per 30 s time intervals. Time intervals were relative to the time of the first PWV recording measured just after cuff deflation.

function. ${ }^{11,12}$ As shown by Naka et al., the brachial-radial PWV decreases in parallel to an increase in the brachial artery diameter during reactive hyperaemia. ${ }^{11}$ Moreover, the authors also demonstrated the impact of NO on PWV changes. Indeed, stimulation of NO release by acetylcholine caused a decrease in PWV along with an increase in the brachial artery diameter, whereas infusion of the NO synthase inhibitor (L-NMMA) caused an increase in PWV and a 
Table 3 FMS per time intervals by sex and occlusion time.

\begin{tabular}{|c|c|c|c|c|c|c|}
\hline & \multicolumn{3}{|l|}{ Gender } & \multicolumn{3}{|l|}{ Occlusion time } \\
\hline & Men $(n=35)$ & Women $(n=36)$ & $P$ value & $3 \min (n=35)$ & $5 \min (n=36)$ & $P$ value \\
\hline \multicolumn{7}{|c|}{ FMS (\%) by time intervals (s)* } \\
\hline First measurement & $-12.7 \pm 12.7$ & $-14.9 \pm 9.78$ & 0.41 & $-9.96 \pm 7.37$ & $-17.6 \pm 13.1$ & 0.0038 \\
\hline $0-30$ & $-9.19 \pm 10.8$ & $-11.6 \pm 9.23$ & 0.31 & $-6.18 \pm 7.22$ & $-14.6 \pm 10.8$ & 0.0003 \\
\hline $30-60$ & $-6.32 \pm 11.4$ & $-8.52 \pm 8.18$ & 0.35 & $-2.58 \pm 7.27$ & $-12.2 \pm 9.92$ & $<0.0001$ \\
\hline $60-90$ & $-4.68 \pm 9.66$ & $-8.82 \pm 8.33$ & 0.059 & $-2.67 \pm 6.96$ & $-10.8 \pm 9.42$ & 0.0001 \\
\hline $90-120$ & $-4.29 \pm 7.60$ & $-5.45 \pm 7.68$ & 0.52 & $-2.35 \pm 7.18$ & $-7.34 \pm 7.28$ & 0.0049 \\
\hline $120-150$ & $-3.08 \pm 7.89$ & $-4.35 \pm 7.49$ & 0.49 & $-1.38 \pm 6.57$ & $-6.01 \pm 8.04$ & 0.0099 \\
\hline $150-180$ & $-3.20 \pm 8.07$ & $-3.18 \pm 7.12$ & 0.99 & $-0.72 \pm 6.45$ & $-5.59 \pm 7.84$ & 0.0056 \\
\hline $180-210$ & $-4.68 \pm 7.57$ & $-4.56 \pm 7.25$ & 0.95 & $-3.48 \pm 5.95$ & $-5.73 \pm 8.44$ & 0.20 \\
\hline $210-240$ & $-3.33 \pm 7.22$ & $-2.80 \pm 6.38$ & 0.75 & $-1.51 \pm 4.92$ & $-4.62 \pm 7.99$ & 0.054 \\
\hline
\end{tabular}

Values are mean $( \pm S D)$. *Time intervals were relative to the time of the first PWV recording measured just after cuff deflation. FMS indicates flow-mediated slowing; PWV, pulse wave velocity.
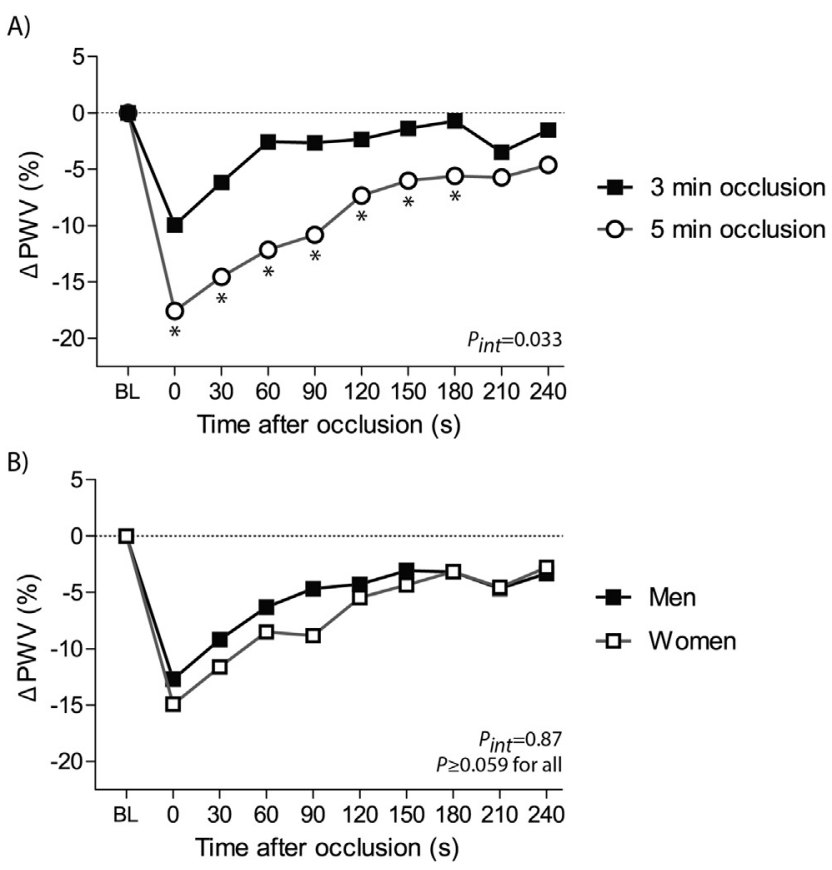

Figure 3 The course of PWV changes by occlusion time (panel A) and sex (panel B). Changes in pulse wave velocity $(\triangle \mathrm{PWV})$ following hyperaemia were averaged per $30 \mathrm{~s}$ intervals, starting from the first measurement after cuff release. ${ }^{*} P<0.05$ for comparison vs 3 -min of occlusion. $\mathrm{BL}$ indicates baseline; FMS, flow-mediated slowing. $P_{\text {int }}$ for interaction between the FMS response throughout all post-occlusion periods and the occlusion time (panel A) or sex (panel B).

decrease in the brachial artery diameter. ${ }^{11}$ This suggests that FMS of PWV during reactive hyperaemia might be used as a marker of arterial distensibility and endothelial function.

In our study, to assess FMS we used paired oscillometric cuffs together with fully automated commercially available software allowing a beat-to-beat assessment of brachialradial PWV at baseline and during a post-occlusion period. In contrast to FMD, the FMS procedure requires little training, time and logistics, promoting its use in large-scale population studies and screening programs.

In our study we demonstrated that the magnitude of FMS is related to the duration of the occlusion period. Previous studies examined the effect of suprasystolic occlusion time on the vasodilatory response to reactive hyperaemia. ${ }^{5,17}$ In 11 young healthy subjects, Sinoway et al. did not observe any significant differences in the maximal peak blood flows and changes in the artery diameter following 3-min and 10min of occlusion. ${ }^{17}$ In contrast, an FMS study in 43 healthy volunteers demonstrated that longer occlusion times resulted in greater rates of carotid-radial PWV change early after cuff release. ${ }^{18}$ In line with the latter study, we also observed a significantly greater PWV change after a longer occlusion time (5-min) as compared to a shorter occlusion period (3-min). Thus, similarly to recommendations for FMD testing, ${ }^{5,16}$ our findings confirm the use of a suprasystolic occlusion period of at least 5-min for vasomotor function assessment by FMS.

Age-associated changes in vascular wall properties are characterised by intima-media thickening, loss of elastic properties, and, therefore, stiffening of the arterial wall. ${ }^{19}$ Overall, these pathological processes gradually hamper the vasodilatory capacity of the conduit arteries to adapt to increased shear stress. In line with our findings, previous studies showed an age-related deterioration in vasoreactivity as assessed by the FMD of the brachial artery ${ }^{20}$ or the FMS of carotid-radial PWV. ${ }^{21}$ In addition, our study explored the association of age with the FMS of PWV of the brachial artery during each $30 \mathrm{~s}$ interval of the postocclusion period. We observed that the FMS of PWV at early post-occlusion periods (30-90 s) was significantly correlated with age, whereas the $\triangle P W V$ at the later postocclusion periods appeared unaffected by age.

It is well known that vasomotor function is affected by cardiovascular factors such as hypertension and smoking. ${ }^{22-24}$ Indeed, high blood pressure and exposure to cigarette smoke damage the endothelial lining of vascular smooth muscle cells through enhanced oxidative stress. $^{22-24}$ The Framingham Heart Study showed in 2883 randomly recruited participants that both systolic blood pressure and current smoking were independent 
Table 4 Correlates of FMS as selected by stepwise regression.

\begin{tabular}{|c|c|c|c|c|c|c|c|c|c|}
\hline \multirow[t]{2}{*}{ Parameters } & \multicolumn{9}{|c|}{$\Delta \mathrm{PWV}(\%)$ by time intervals $(\mathrm{s})^{*}$} \\
\hline & $\begin{array}{l}\text { First } \\
\text { measurement }\end{array}$ & $0-30 \mathrm{~s}$ & $30-60 \mathrm{~s}$ & $60-90 \mathrm{~s}$ & $90-120 \mathrm{~s}$ & $120-150 \mathrm{~s}$ & $150-180 \mathrm{~s}$ & $180-210 \mathrm{~s}$ & $210-240 s$ \\
\hline Adjusted $\mathrm{R}^{2}(\%)$ & 36.8 & 32.7 & 35.4 & 36.4 & 29.1 & 29.3 & 26.8 & 9.40 & 23.8 \\
\hline \multicolumn{10}{|c|}{ Occlusion time (5 vs 3 min) } \\
\hline$\beta \pm \mathrm{SE}$ & $-9.29 \pm 2.10$ & $-9.32 \pm 2.01$ & $-9.63 \pm 1.99$ & $-7.90 \pm 1.92$ & $-5.32 \pm 1.66$ & $-4.23 \pm 1.67$ & $-4.74 \pm 1.66$ & $-1.83 \pm 0.86$ & $-4.36 \pm 1.53$ \\
\hline$P$ value & $<0.0001$ & $<0.0001$ & $<0.0001$ & 0.0001 & 0.0022 & 0.014 & 0.0059 & 0.037 & 0.0060 \\
\hline Partial R ${ }^{2}(\%)$ & 19.4 & 21.5 & 27.2 & 23.7 & 15.5 & 12.9 & 13.1 & 6.11 & 7.11 \\
\hline \multicolumn{10}{|c|}{ Age $(+10 \text { years })^{* *}$} \\
\hline$\beta \pm \mathrm{SE}$ & $3.68 \pm 0.77$ & $2.99 \pm 0.74$ & $2.10 \pm 0.73$ & $1.23 \pm 0.69$ & $0.83 \pm 0.62$ & $0.041 \pm 0.60$ & $-0.084 \pm 0.60$ & $0.76 \pm 0.63$ & $0.98 \pm 0.57$ \\
\hline$P$ value & $<0.0001$ & 0.0001 & 0.0053 & 0.079 & 0.18 & 0.95 & 0.89 & 0.23 & 0.092 \\
\hline Partial R ${ }^{2}(\%)$ & 17.4 & 11.2 & 4.67 & 3.38 & - & - & - & - & - \\
\hline \multicolumn{10}{|l|}{ Female $(0,1)$} \\
\hline $\begin{array}{l}\beta \pm \mathrm{SE} \\
P \text { value }\end{array}$ & - & - & - & $\begin{array}{l}-4.00 \pm 1.88 \\
0.037\end{array}$ & - & - & - & - & - \\
\hline Partial R ${ }^{2}(\%)$ & & & & 7.56 & & & & & \\
\hline \multicolumn{10}{|c|}{ DBP, (+10 mm Hg) } \\
\hline $\begin{array}{l}\beta \pm \mathrm{SE} \\
P \text { value }\end{array}$ & - & - & - & - & $\begin{array}{l}-1.56 \pm 0.85 \\
0.074\end{array}$ & $\begin{array}{l}-1.97 \pm 0.87 \\
0.027\end{array}$ & $\begin{array}{l}-2.32 \pm 0.85 \\
0.0087\end{array}$ & $\begin{array}{l}-2.04 \pm 0.92 \\
0.031\end{array}$ & $\begin{array}{l}-2.32 \pm 0.79 \\
0.0048\end{array}$ \\
\hline Partial R ${ }^{2}(\%)$ & & & & & 3.41 & 5.44 & 7.35 & 4.61 & 9.03 \\
\hline \multicolumn{10}{|l|}{ Smoking $(0,1)$} \\
\hline $\begin{array}{l}\beta \pm S E \\
P \text { value }\end{array}$ & - & - & - & $\begin{array}{l}-5.36 \pm 2.80 \\
0.060\end{array}$ & $\begin{array}{l}-4.05 \pm 2.30 \\
0.083\end{array}$ & $\begin{array}{l}-7.33 \pm 2.34 \\
0.0026\end{array}$ & $\begin{array}{l}-5.81 \pm 2.30 \\
0.014\end{array}$ & - & $\begin{array}{l}-4.76 \pm 2.11 \\
0.028\end{array}$ \\
\hline Partial R ${ }^{2}(\%)$ & & & & 3.54 & 3.29 & 5.75 & 4.61 & & 4.64 \\
\hline \multicolumn{10}{|c|}{ oxLDL, per doubling } \\
\hline $\begin{array}{l}\beta \pm \mathrm{SE} \\
P \text { value }\end{array}$ & - & - & - & $\begin{array}{l}-1.31 \pm 0.62 \\
0.040\end{array}$ & $\begin{array}{l}-1.41 \pm 0.53 \\
0.0098\end{array}$ & $\begin{array}{l}-1.39 \pm 0.53 \\
0.011\end{array}$ & $\begin{array}{l}-1.30 \pm 0.53 \\
0.016\end{array}$ & - & - \\
\hline Partial R ${ }^{2}(\%)$ & & & & 2.94 & 7.11 & 6.37 & 6.68 & & \\
\hline \multicolumn{10}{|c|}{ History of DM $(0,1)$} \\
\hline $\begin{array}{l}\beta \pm \mathrm{SE} \\
P \text { value }\end{array}$ & - & - & - & - & - & - & - & - & $\begin{array}{l}-5.08 \pm 2.29 \\
0.030\end{array}$ \\
\hline Partial R ${ }^{2}(\%)$ & & & & & & & & & 5.70 \\
\hline
\end{tabular}




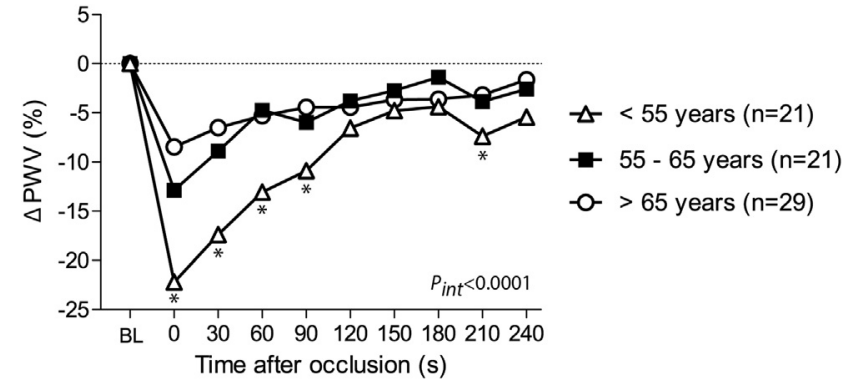

Figure 4 The course of PWV changes by age tertiles. Changes in pulse wave velocity $(\triangle \mathrm{PWV})$ following hyperaemia were averaged per $30 \mathrm{~s}$ intervals, starting from the first measurement after cuff release. ${ }^{*} P<0.05$ for comparison with subjects $>55$ years of age. BL indicates baseline; FMS, flow-mediated slowing. $P_{\text {int }}$ for interaction between the FMS response throughout all post-occlusion periods and age groups.

determinants of a lower peak FMD. ${ }^{20}$ In our small community-based sample, however, cardiovascular risk factors were not independently correlated with the early peak FMS of PWV of the brachial artery. On the other hand, we observed that subjects with high level of diastolic blood pressure and oxidized LDL as well as smokers exhibited a delay in recovery of PWV following hyperaemia as compared to a healthy reference group. To our knowledge, our study is the first to report the $\triangle P W V$ continuously including the later stage of post-occlusion. In our healthy reference subjects the hyperaemia-mediated $\mathrm{PWV}$ returned to baseline PWV values within 3-min after deflation (180 s) which is similar to the time reported previously on FMD recovery in healthy subjects. ${ }^{25}$ In contrast, in subjects with cardiovascular risk factors the artery might be slower to return to baseline and, therefore, a longer period of time would be required for a full PWV recovery. Future studies should further clarify the mechanisms underlying this observation.

This study has to be interpreted within the context of its potential limitations. First, considering the relative small sample size, our findings should be validated in a larger cohort of randomly recruited subjects. Second, as previous FMS studies did not address our objectives, we could only interpret our findings in the light of FMD studies or reports on hyperaemia-mediated changes in carotid-toradial PWV. Furthermore, the cross-sectional design of our study did not allow inferring causality. Finally, our study population only included white European participants, limiting the generalizability of our findings to other ethnicities.

In conclusion, in our population study we confirmed the preference of using a 5-min occlusion time for the assessment of vasomotor function by FMS. Whereas the early peak of FMS might deteriorate with ageing, cardiovascular risk factors might affect the time of recovery of PWV following reactive hyperaemia. Our work demonstrates the potential value of continuous measurement of changes in PWV of the brachial artery during post-occlusion period. Future studies should further investigate whether the FMS response might help in risk stratification of patients.

A) Unadjusted FMS response
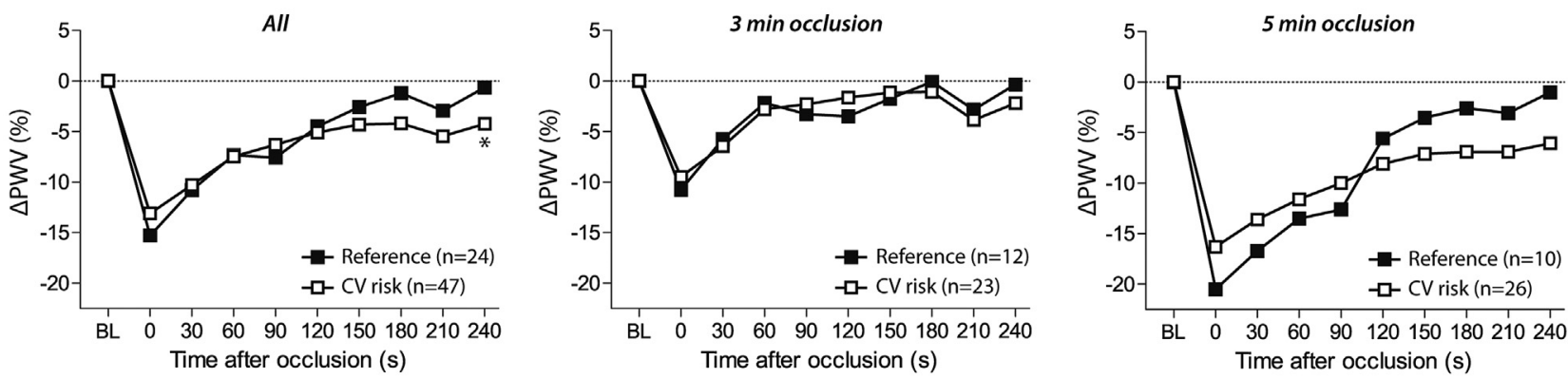

B) Age-adjusted FMS response
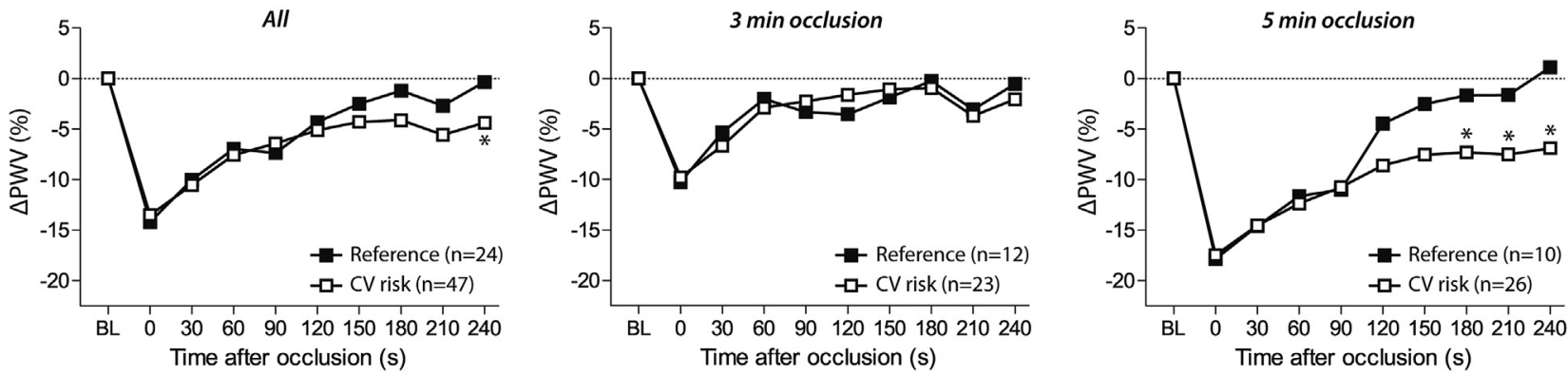

Figure 5 The course of PWV changes by cardiovascular risk pattern. Changes in pulse wave velocity ( $\triangle P W V$ ) following hyperaemia are presented as unadjusted $(A)$ or age-adjusted values $(B)$. ${ }^{*} P<0.05$ for comparison with reference group including non-smoking normotensives. BL indicates baseline; FMS, flow-mediated slowing. 


\section{Funding}

The Studies Coordinating Centre also received grants from the Fonds voor Wetenschappelijk Onderzoek Vlaanderen, Brussels, Belgium (grants G.0880.13, G. 0881.13 and $11 \mathrm{Z0916N).}$

\section{Conflict of interest}

None.

\section{Appendix A. Supplementary data}

Supplementary data related to this article can be found at https://doi.org/10.1016/j.artres.2017.12.001.

\section{References}

1. Cahill PA, Redmond EM. Vascular endothelium - gatekeeper of vessel health. Atherosclerosis 2016;248:97-109.

2. Flammer AJ, Anderson T, Celermajer DS, Creager MA, Deanfield J, Ganz P, et al. The assessment of endothelial function: from research into clinical practice. Circulation 2012;126(6):753-67.

3. Mordi I, Mordi N, Delles C, Tzemos N. Endothelial dysfunction in human essential hypertension. J Hypertens 2016;34(8): 1464-72.

4. Nohria A, Gerhard-Herman M, Creager MA, Hurley S, Mitra D, Ganz P. Role of nitric oxide in the regulation of digital pulse volume amplitude in humans. J Appl Physiol 2006;101(2):545-8.

5. Corretti MC, Anderson TJ, Benjamin EJ, Celermajer D, Charbonneau F, Creager MA, et al. Guidelines for the ultrasound assessment of endothelial-dependent flow-mediated vasodilation of the brachial artery: a report of the International Brachial Artery Reactivity Task Force. J Am Coll Cardiol 2002;39(2):257-65.

6. Faulx MD, Wright AT, Hoit BD. Detection of endothelial dysfunction with brachial artery ultrasound scanning. Am Heart J 2003;145(6):943-51.

7. Kuvin JT, Patel AR, Sliney KA, Pandian NG, Sheffy J, Schnall RP, et al. Assessment of peripheral vascular endothelial function with finger arterial pulse wave amplitude. Am Heart J 2003; 146(1):168-74.

8. Hamburg NM, Keyes MJ, Larson MG, Vasan RS, Schnabel R, Pryde MM, et al. Cross-sectional relations of digital vascular function to cardiovascular risk factors in the Framingham heart study. Circulation 2008;117(19):2467-74.

9. Allen J. Photoplethysmography and its application in clinical physiological measurement. Physiol Meas 2007;28(3):R1-39.

10. Kuznetsova T, Van Vlierberghe E, Knez J, Szczesny G, Thijs L, Jozeau $D$, et al. Association of digital vascular function with cardiovascular risk factors: a population study. BMJ Open 2014; 4(3), e004399.
11. Naka KK, Tweddel AC, Doshi SN, Goodfellow J, Henderson AH. Flow-mediated changes in pulse wave velocity: a new clinical measure of endothelial function. Eur Heart J 2006;27(3): 302-9.

12. Ellins EA, New KJ, Datta DBN, Watkins S, Haralambos K, Rees $A$, et al. Validation of a new method for non-invasive assessment of vasomotor function. Eur J Prev Cardiol 2016; 23(6):577-83.

13. McDonald DA. Regional pulse-wave velocity in the arterial tree. J Appl Physiol 1968;24(1):73-8.

14. Cavalcante JL, Lima JAC, Redheuil A, Al-Mallah MH. Aortic stiffness: current understanding and future directions. J Am Coll Cardiol 2011;57(14):1511-22.

15. Ramsey MW, Goodfellow J, Jones CJ, Luddington LA, Lewis MJ, Henderson $\mathrm{AH}$. Endothelial control of arterial distensibility is impaired in chronic heart failure. Circulation 1995;92(11): 3212-9.

16. Thijssen DHJ, Black MA, Pyke KE, Padilla J, Atkinson G, Harris RA, et al. Assessment of flow-mediated dilation in humans: a methodological and physiological guideline. Am J Physiol Heart Circ Physiol 2011;300(1):H2-12.

17. Sinoway LI, Hendrickson C, Davidson WRJ, Prophet S, Zelis R. Characteristics of flow-mediated brachial artery vasodilation in human subjects. Circ Res 1989;64(1):32-42.

18. Torrado J, Farro I, Bia D, Zocalo Y, Valls G, Valero M, et al. Levels and rates of change in carotid-radial pulse wave velocity associated with reactive hyperaemia: analysis of the dependence on transient ischemia length. Conf Proc. Annu Int Conf IEEE Eng Med Biol 2010;2010:2865-8.

19. Harvey A, Montezano AC, Lopes RA, Rios F, Touyz RM. Vascular fibrosis in aging and hypertension: molecular mechanisms and clinical implications. Can J Cardiol 2016;32(5):659-68.

20. Benjamin EJ, Larson MG, Keyes MJ, Mitchell GF, Vasan RS, Keaney JFJ, et al. Clinical correlates and heritability of flowmediated dilation in the community: the Framingham heart study. Circulation 2004;109(5):613-9.

21. Kamran H, Salciccioli L, Venkatesan B, Namana V, Kumar P, Pushilin S, et al. Determinants of a blunted carotid-to-radial pulse wave velocity decline in response to hyperemia. Angiology 2010;61(6):591-4.

22. Messner B, Bernhard D. Smoking and cardiovascular disease: mechanisms of endothelial dysfunction and early atherogenesis. Arterioscler Thromb Vasc Biol 2014;34(3):509-15.

23. Hurtubise J, McLellan K, Durr K, Onasanya O, Nwabuko D, Ndisang JF. The different facets of dyslipidemia and hypertension in atherosclerosis. Curr Atherosclerosis Rep 2016; 18(12):82.

24. Steffen $Y$, Vuillaume $G$, Stolle $K$, Roewer $K$, Lietz $M$, Schueller J, et al. Cigarette smoke and LDL cooperate in reducing nitric oxide bioavailability in endothelial cells via effects on both eNOS and NADPH oxidase. Nitric Oxide Biol Chem 2012;27(3):176-84.

25. Barton M, Turner AT, Newens KJ, Williams CM, Thompson AK. Minimum recovery time between reactive hyperemia stimulus in the repeated measurement of brachial flow-mediated dilatation. Ultrasound Med Biol 2011;37(6):879-83. 